TUM-HEP 215/95

IC $/ 95 / 29$

hep-ph/9503233

February 1995

\title{
Axino Mass in Supergravity Models
}

\author{
E. J. Chun* \\ International Center for Theoretical Physics \\ P. O. Box 586, 34100 Trieste, Italy \\ and \\ A. Lukas ${ }^{\dagger}$ \\ Physik Department \\ Technische Universität München \\ D-85747 Garching, Germany \\ and \\ Max-Planck-Institut für Physik \\ Werner-Heisenberg-Institut \\ P. O. Box 4012 12, Munich, Germany
}

\begin{abstract}
We analyze the mass of the axino, the fermionic superpartner of the axion, in general supergravity models incorporating a Peccei-Quinn-symmetry and determine the cosmological constraints on this mass. In particular, we derive a simple criterion to identify models with an LSP-axino which has a mass of $O\left(m_{3 / 2}^{2} / f_{P Q}\right)=O(\mathrm{keV})$ and can serve as a candidate for (warm) dark matter. We point out that such models have very special properties and in addition, the small axino mass has to be protected against radiative corrections by demanding small couplings in the Peccei-Quinn-sector. Generically, we find an axino mass of order $m_{3 / 2}$. Such masses are constrained by the requirement of an axino decay which occurs before the decoupling of the ordinary LSP. Especially, for a large Peccei-Quinn-scale $f_{P Q}>10^{11} \mathrm{GeV}$ this constraint might be difficult to fulfill.
\end{abstract}

\footnotetext{
*Email:chun@ictp.trieste.it

†Email:alukas@physik.tu-muenchen.de
} 
The implications of axions have been examined extensively since their existence was suggested by an attractive mechanism for resolving the strong CP problem [1, 2, 3]. Even though axions are very weakly interacting, their astrophysical and cosmological effects are strong enough to narrow down the window of the Peccei-Quinn-scale $f_{P Q}$ to $10^{10} \mathrm{GeV}<f_{P Q}<$ $10^{12} \mathrm{GeV}$ [5]. On the same footing the axino as the supersymmetric partner of the axion can play an important role in astrophysics and cosmology [6]. An interesting feature is that axinos may receive a mass of order $\mathrm{keV}$ which would render them a good candidate for warm dark matter. If axinos are heavier than a few keV they have to decay fast enough not to upset any standard prediction of big-bang cosmology. Given the weakness of their interactions, a constraint on their lifetime put a rather severe limit on the lower bound of their mass. Therefore it is very important to know the axino mass in discussing the cosmological implications of supersymmetric axion models.

In global supersymmetry (SUSY) the calculation of the axino mass was performed in refs. [7, 8]. In this paper, we will provide the computations in models with local supersymmetry (supergravity). Some partial results have been obtained in refs. [9, 10]. In the case of spontaneously-broken global SUSY the axino mass is of the order $m_{3 / 2}^{2} / f_{P Q} \sim \mathrm{keV}$ where $m_{3 / 2}(\lesssim 1 \mathrm{TeV})$ is taken to be the global SUSY-breaking scale [7, 8]. On the contrary, in the context of supergravity, the axino mass can be of order $m_{3 / 2}$ as first noticed in ref. [9]. Soon after this it was realized that the axino mass is truly model-dependent and the global SUSY value $m_{3 / 2}^{2} / f_{P Q}$ may be obtained in supergravity models as well [10. We will extend those results in a generic treatment of supergravity models also including radiative corrections.

The prime motivation for supergravity is well-known. Realistic supersymmetric generalizations of the standard model are based on local SUSY spontaneously broken in a so-called hidden sector at a mass scale of order $M_{S} \sim 10^{11} \mathrm{GeV}[12]$. The induced SUSY breaking scale in the observable sector is determined by a value of the order of the gravitino mass $m_{3 / 2} \sim M_{S}^{2} / M_{P}$ where $M_{P}$ is the Planck scale. Axionic extensions of the minimal supersymmetric standard model (MSSM) inevitably incorporate an extra sector which provides spontaneous breaking of the Peccei-Quinn $U(1)$-symmetry at the scale $f_{P Q}$. This sector (PQ-sector) is considered as a part of the observable sector. In the framework of effective supergravity theories with a Lagrangian composed out of a global SUSY part and soft terms the hidden sector dependences are encoded in the soft terms. We will rely mostly on this effective approach as it makes the calculations tractable.

A color anomaly in the PQ-sector can be introduced in two ways. The fields $S$ in this sector can be coupled to the standard Higgs doublets $H_{1}, H_{2}$ of the MSSM like $g S H_{1} H_{2}$

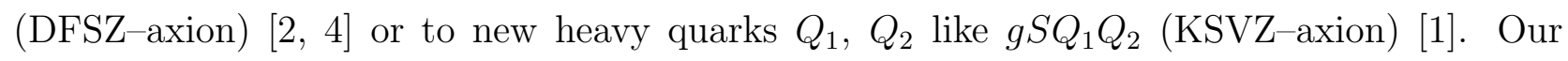
analysis of axino mass will be concerned with the tree level result in the effective theory which is obtained after breaking the PQ-symmetry and SUSY and should therefore not depend on 
which implementation of the axion is chosen. We will find that this mass crucially depends on the structure of the PQ-sector as well as on the hidden sector. It should, however, be mentioned that in addition the above couplings and the corresponding trilinear soft terms lead to a one-loop radiative mass of order $m_{\tilde{a} \text {,loop }} \simeq 3 g^{2} A m_{3 / 2} / 16 \pi^{2}$ ( $A$ is the trilinear soft coupling) [11, 9]. For the DFSZ-axion this contribution is clearly negligible since the coupling $g$ has to be quite small $\left(g \sim 10^{-7}\right)$ in order to generate reasonable Higgs-masses. In the KSVZ-implementation there is no such restriction on $g$ and the above one-loop contribution can modify the result for the axino mass to be obtained below.

An interesting observation is that the axino mass depends on whether the PQ-sector admits additional accidental zero modes on the global SUSY level. As a general statement we find that the axino mass cannot be bigger than $O\left(m_{3 / 2}\right)$ if the axion mode is the only global zero mode in the PQ-sector. In order to see this, it is useful to analyze the full supergravity Lagrangian. Later, we will confirm this result by using the effective Lagrangian approach.

The PQ-sector consists of an arbitrary number of singlets $S^{a}$ with charge $q_{a}$ under the PQ-symmetry and provides its spontaneous breaking at the scale $f_{P Q}=\left(\sum_{a} q_{a}^{2}\left|v^{a}\right|^{2}\right)^{1 / 2}$, where $v^{a}=\left\langle s^{a}\right\rangle$. The axion multiplet $\Phi$ is given by $\Phi=\sum_{a} q_{a} v^{a} S^{a} / f_{P Q}$. Its component field content $\operatorname{reads} \Phi \sim(s+i a, \tilde{a})$ with the axion $a$, the saxion $s$ and the axino $\tilde{a}$. Supersymmetry is broken mainly by the hidden sector with singlet fields $Z^{i}$. To simplify the argument we assume minimal kinetic term for the PQ fields as well as for the hidden sector fields. The scalar potential reads

$$
V=M_{P}^{2} \exp \left[G / M_{P}^{2}\right]\left(\sum_{i} G_{i} G_{i}^{*}+\sum_{a} G_{a} G_{a}^{*}-3 M_{P}^{2}\right),
$$

where $G=K+M_{P}^{2} \ln \left|W / M_{P}^{3}\right|^{2}$ and $W=W\left(S^{a}\right)+W\left(Z^{i}\right)$. Spontaneous supersymmmetry breaking implies $\left\langle G_{i}\right\rangle \simeq M_{P}$ for some $i$ and generation of a gravitino mass, $m_{3 / 2} \simeq$ $M_{P} \exp \left[G / 2 M_{P}^{2}\right]$. We also have to admit the possibility that $\left\langle G_{a}\right\rangle \lesssim M_{P}$ for some $a$. We now look at the minimization condition $\left\langle V_{i}\right\rangle=0$ to estimate the axino mass. Vanishing of the cosmological constant $\langle V\rangle=0$ is assumed. Then we have

$$
\left\langle V_{a}\right\rangle=\left\langle G_{a b} G_{b}^{*}+G_{a i} G_{i}^{*}+G_{a}^{*}\right\rangle .
$$

If we take a massive mode $S^{a}$ in the PQ-sector, $\left\langle G_{a b}\right\rangle$ is dominated by

$$
\left\langle G_{a b}\right\rangle \simeq\left\langle M_{P}^{2} \frac{W_{a b}}{W}\right\rangle \simeq \frac{f_{P Q}}{m_{3 / 2}} \delta_{a b},
$$

since $\langle W\rangle \simeq M_{P}^{2} m_{3 / 2}$. In addition, we can estimate the maximal order of magnitude of $\left\langle G_{a i}\right\rangle$

$$
\left\langle G_{a i}\right\rangle \simeq\left\langle-M_{P}^{2} \frac{W_{a} W_{i}}{W^{2}}\right\rangle \simeq \frac{f_{P Q}^{2}}{M_{P} m_{3 / 2}}
$$


since $\left\langle W_{i}\right\rangle \simeq M_{P} m_{3 / 2}$ maximally and $\left\langle W_{a}\right\rangle \lesssim f_{P Q}^{2}$. Therefore the condition $\left\langle V_{a}\right\rangle=0$ gives $\left\langle G_{a}\right\rangle \lesssim f_{P Q}$. In the axion direction $G_{\Phi}=\sum_{a} q_{a} v^{a} G_{a} / f_{P Q}$, we also have $\left\langle G_{\Phi}\right\rangle=$ $\left\langle\sum_{a} q_{a} v^{a} K_{a} / f_{P Q}\right\rangle=\sum_{a} q_{i}\left|v^{a}\right|^{2} / f_{P Q} \lesssim f_{P Q}$. Since the absence of accidental zero modes is required in the $\mathrm{PQ}$-sector one finds that $\left\langle G_{a}\right\rangle \lesssim f_{P Q}$ for all the fields $S^{a}$.

It is now straightforward to estimate the maximal order of the axino mass by using the fermion mass matrix in supergravity models, $\mathcal{M}_{i j}=M_{P} \exp \left(G / 2 M_{P}^{2}\right)\left[G_{a b}+G_{a} G_{b} / M_{P}\right]$. Along the axino direction,

$$
\mathcal{M}_{\Phi b} \simeq m_{3 / 2}\left\langle\sum_{a} \frac{q_{a} v^{a}}{f_{P Q}} G_{a b}-\sum_{a} \frac{q_{a} v^{a} G_{a}}{f_{P Q}} \frac{G_{b}}{M_{P}}\right\rangle .
$$

From the $U(1)$ invariance of $G$, we get $\sum_{a} q_{a} v a\left\langle G_{a b}\right\rangle=q_{b}\left(v_{b}-\left\langle G_{b}\right\rangle\right) \lesssim f_{P Q}$. Hence the axino mass is maximally of order $m_{3 / 2}$.

The above order-of-magnitude estimation is indeed insensitive to the specific forms of the kinetic term or the superpotential as long as the hidden sector fields have only nonrenormalizable couplings to the observable sector. This happens because higher power terms in $K$ or $W$ are naturally suppressed by powers of $M_{P}$ which renders their contribution negligible. Therefore we conclude that the axino mass in general supergravity models is at most of the order $m_{3 / 2}$ if no other zero mode than the axion is present in the $\mathrm{PQ}$-sector.

On the other hand if there are extra zero modes we are not able to constrain further the order of $\left\langle G_{a}\right\rangle$ for a zero mode direction $a$ so that the above argumentation breaks down. In fact, axino masses $\gg m_{3 / 2}$ are possible in those models as we will see below.

We will now calculate the actual value of the axino mass relying on the effective supergravity Lagrangian with arbitrary soft terms. This allows to consider axino masses down to $O\left(m_{3 / 2}^{2} / f_{P Q}\right)$ where the next to leading order in the $1 / M_{P}$-expansion of supergravity becomes important.

The superpotential $W$ of the PQ-sector is expanded as

$$
W=f_{a} S^{a}+\frac{1}{2} f_{a b} S^{a} S^{b}+\frac{1}{6} f_{a b c} S^{a} S^{b} S^{c}
$$

and a departure from standard soft terms is encoded in

$$
N=d_{a} f_{a} s^{a}+\frac{1}{2} d_{a b} f_{a b} s^{a} s^{b}+\frac{1}{6} d_{a b c} f_{a b c} s^{a} s^{b} s^{c} .
$$

Then the scalar potential reads

$$
V=\bar{\partial}^{b} \bar{W} \partial_{b} W+m_{c}^{2 b} \bar{s}_{b} s^{c}+m_{3 / 2}\left[s^{b} \partial_{b} W+(A-3) W+N+\text { h.c. }\right] .
$$

To minimize this potential we apply the following strategy : The VEVs $v^{a}$ are split into a global SUSY value $u^{a}$ with $\partial_{a} W(u)=0$ and corrections $w^{a}$ due to the soft terms, $v^{a}=u^{a}+w^{a}$. 
Expanding $\partial_{a} V$ around the global minimum $\left(u^{a}\right)$ results in

$$
\begin{aligned}
\left(\partial_{a} V\right)(v)= & \frac{1}{2} f_{a b e} \bar{f}^{b c d} \bar{w}_{c} \bar{w}_{d} w^{e}+\frac{1}{2} M_{a b} \bar{f}^{b c d} \bar{w}_{c} \bar{w}_{d}+\bar{M}^{b c} f_{a b e} \bar{w}_{c} w^{e} \\
& +\frac{m_{3 / 2}}{2}\left(A+d_{a b c}\right) f_{a b c} w^{b} w^{c}+m_{3 / 2}\left[\left((A-1) M_{a b}+d_{a b} f_{a b}\right) w^{b}\right. \\
& \left.+\left(1+d_{a b c}\right) f_{a b c} u^{b} w^{c}\right]+m_{a}^{2 b} \bar{w}_{b}+M_{a b} \bar{M}^{b c} \bar{w}_{c}+I_{a}
\end{aligned}
$$

with the global mass matrix

$$
M_{a b}=\partial_{a} \partial_{b} W(u)
$$

and the definitions

$$
I_{a}=m_{3 / 2} J_{a}+m_{a}^{2 b} \bar{u}_{b}, \quad J_{a}=M_{a b} u^{b}+\left(\partial_{a} N\right)(u)
$$

Care should be taken on the choice of $\left(u^{a}\right)$. With any global minimum also a rotation $u^{a} \rightarrow$ $\exp \left(q_{a} z\right) u^{a}$ with $z=x+i y$ under the complexified $U_{P Q}(1)$ leads to such a minimum. Despite the $y$-dependent part of this symmetry which is clearly present in the full theory the $x^{-}$ dependent part (present because of the holomorphy of the superpotential) is broken by the soft terms. Therefore an appropriate fixing for the $x$-dependent part of the symmetry should be applied such that the global minimum comes close to the local values $\left(v^{a}\right)$ resulting in small expansion coefficients $\left(w^{a}\right)$. Such a fixing is provided by the condition $w_{\alpha}=\sum_{a} q_{a} u^{a} w^{a} / f_{P Q}=$ 0 implying that the correction in the global axion direction (denoted by an index $\alpha$ ) vanishes.

As can be expected the axino mass is expressible in terms of the corrections $\left(w^{a}\right)$ :

$$
\begin{aligned}
(\mathcal{M} \tilde{a})_{a} & =-\frac{1}{f_{P Q}} q_{a}^{b}\left(\partial_{b} W\right)(v) \\
& =-\frac{1}{f_{P Q}} q_{a}^{b}\left(M_{b c} w^{b}+\frac{1}{2} f_{b c d} w^{c} w^{d}+\cdots\right) .
\end{aligned}
$$

These corrections have to be determined from eq. (9). Let us work in a basis with diagonal global mass matrix $M_{a b}=M_{a} \delta_{a b}$. We denote massive modes with indices $i, j, \cdots$ and possible additional zero modes with indices $\beta, \gamma, \cdots$. Then an important observation is that the corrections $w_{i}$ are basically determined by the linear term $\left|M_{i}\right|^{2} w^{i}$ and $I_{i}$ in eq. (9). In zero mode directions the situation might be more complicated since $\left|M_{\beta}\right|^{2} w^{\beta}$ can be small compared to other terms in eq. (9). Taking this into account we conclude that the expression

$$
(\mathcal{M} \tilde{a})_{a} \simeq q_{a}^{i} \frac{\bar{J}_{i}}{f_{P Q} \bar{M}_{i}} m_{3 / 2}-\frac{1}{f_{P Q}} q_{a}^{\beta} M_{\beta} w^{\beta}-\frac{1}{2 f_{P Q}} q_{a}^{\rho} f_{\rho \beta \gamma} w^{\beta} w^{\gamma}+O\left(m_{3 / 2}^{2} / f_{P Q}\right)
$$

gives the correct order of the axino mass. For the moment we leave the values of $w_{\beta}$ unspecified. Instead we concentrate on the first term in eq. (13) and split the expression $J_{a}$ into its Planck 
(or GUT)-scale value $J_{a}^{(0)}$ and corrections $J_{a}^{(1)}$ arising from renormalization down to $f_{P Q}$. The generic value of the corrections can be roughly estimated as

$$
\begin{array}{r}
J_{a}^{(1)}=\lambda_{a}^{\prime} k_{a} f_{P Q}^{2} \\
k_{a} \sim \frac{\lambda_{a}^{2}}{32 \pi^{2}} \ln \left(\frac{M_{P}}{f_{P Q}}\right)
\end{array}
$$

with appropriate combinations $\lambda_{a}, \lambda_{a}^{\prime}$ of the superpotential couplings. According to eq. (11) $J_{a}^{(0)}$ is naively of the order $f_{P Q}^{2}$. Therefore a necessary condition for the axino mass to be much smaller than $m_{3 / 2}$ is that $J_{a}^{(0)}=0$.

In general this condition implies a relation between the structure of the superpotential and the soft terms. It is instructive to analyze this relation for a certain subclass of models, namely those with independent soft coupling $A, B, C$ for the trilinear, bilinear and linear terms in the superpotential, respectively. A computation leads to

$$
J_{a}^{(0)}=(B-C) M_{a b} u^{b}+\frac{1}{2}(A-2 B+C) f_{a b c} u^{b} u^{c} .
$$

Depending on the properties of the superpotential (and assuming that at least one coupling $f_{a}$ is nonzero to force the symmetry breaking) several cases can be distinguished :

- $f_{a b} u^{b}, f_{a b c} u^{b} u^{c}, M_{a b} u^{b} \neq 0$ : Then $J_{a}^{(0)}=0$ if and only if $A=B=C$. No special property of the superpotential is required.

- $f_{a b} u^{b}=0, M u \neq 0$ : Then $J_{a}^{(0)}=0$ if and only if $A=C$. A simple superpotential which fulfills this requirement is e. g. $W=\lambda\left(S S^{\prime}-\mu^{2}\right) Y$ since all $f_{a b}=0$.

- $f_{a b c} u^{b} u^{c}=0, M u \neq 0$ : Then $J_{a}^{(0)}=0$ if and only if $B=C$.

- $M u=0, f_{a b} u^{b} \neq 0$ : Then $J_{a}^{(0)}=0$ if and only if $A-2 B+C=0$. As the only ones these models allow for the full standard pattern $B=A-1, C=A-2$. They possess, however, at least one additional zero mode $\sum_{a} u^{a} S^{a}$. An example is provided by the superpotential $W=\lambda\left(S S^{\prime}-Z^{2}\right) Y-\lambda^{\prime}(Z-\mu)^{3}$ [10].

Observe that in particular for $A=B=C$ the expression $J_{a}^{(0)}$ vanishes in any model.

If no additional zero mode is present the axino mass is already completely determined by the first term in eq. (13). On tree level this means

$$
\begin{aligned}
& J_{a}^{(0)}=0 \quad \leftrightarrow \quad m_{\tilde{a}}^{(0)}=O\left(m_{3 / 2}^{2} / f_{P Q}\right) \\
& J_{a}^{(0)} \neq 0 \quad \leftrightarrow \quad m_{\tilde{a}}^{(0)}=O\left(m_{3 / 2}\right)
\end{aligned}
$$

We have therefore found a simple criterion to decide about the magnitude of the axino mass which for soft terms specified by the couplings $A, B, C$ singles out the particularly simple 
patterns listed above. We remark that the small axino mass in no-scale models observed in ref. [9] can also be understood in terms of our analysis since in those models $A=B=C=0$. A full supergravity computation of the axino mass in no-scale models shows that their treelevel mass even vanishes. Therefore the first line of eq. (17) has to be understood as a generic result which in certain special cases might be too large. In the first case of a light axino mass radiative corrections to the potential parameters can become important. Using eq. (14) this leads to a contributions of

$$
m_{\tilde{a}} \simeq k_{i} m_{3 / 2}
$$

To keep the order $m_{\tilde{a}}=O\left(m_{3 / 2}^{2} / f_{P Q}\right)$ an upper bound on the couplings has to be required. For $m_{3 / 2} \simeq 10^{2} \mathrm{GeV}$ and $f_{P Q} \simeq 10^{11} \mathrm{GeV}$ this implies $\lambda_{i} \lesssim 10^{-4}$. A systematic way to avoid such small couplings is to consider no-scale models. Since gaugino masses are the only source of SUSY-breaking in those models the standard model singlet fields in the PQ-sector will not receive any radiative soft terms.

We see that without additional zero modes a complete answer can be given. In particular we recover the result $m_{\tilde{a}} \lesssim O\left(m_{3 / 2}\right)$.

If additional zero modes are present the situation becomes more complicated since the corrections $w_{\beta}$ in the zero mode directions can become large. In addition we have to consider that the zero entry $M_{\beta}$ of the mass matrix receives a radiative contribution $M_{\beta}=\tilde{\lambda}^{\prime} \tilde{k}_{\beta} f_{P Q}$ with $\tilde{k}_{\beta}$ in analogy to eq. (15) unless it is protected by an additional continuous or discrete symmetry. Let us discuss some relevant cases. First we discuss a model with $J_{a}^{(0)}=0$, e. g. a model of the last type in the above list for $A, B, C$-type soft terms. Then a tree level axino mass $O\left(m_{3 / 2}^{2} / f_{P Q}\right)$ is not guaranteed as opposed to the case without additional zero modes : If the terms in eq. (9) linear in $w_{\beta}$ vanish (which e. g. occurs for $f_{\beta \gamma}=0, f_{\beta \gamma \delta}=0$ or $A=B$ ) a value $w_{\beta}=O\left(I_{\beta}^{1 / 3}\right)$ results which causes an axino mass given by $m_{\tilde{a}}^{(0)}=O\left(\left(m_{3 / 2} / f_{P Q}\right)^{1 / 3} m_{3 / 2}\right)$. In any case a small axino mass $\ll m_{3 / 2}$ has to be stabilized against radiative corrections. For superpotential couplings $\lambda=O(1)$ the axino mass is shifted to $m_{\tilde{a}}=O\left(m_{3 / 2}\right)$.

Now we assume that $J_{a}^{(0)} \neq 0$. Then for values $\lambda=O(1)$ the linear term $\left|M_{\beta}\right|^{2} \bar{w}_{\beta}$ in eq. (9) will dominate and the axino mass is given by

$$
m_{\tilde{a}}=O\left(m_{3 / 2} / \tilde{k}_{\beta}\right) .
$$

If, on the other hand, the couplings $\lambda$ are very small we can have $w_{\beta}=O\left(I_{\beta}^{1 / 3}\right)$ leading to an axino mass $m_{\tilde{a}}=O\left(\left(m_{3 / 2}^{2} f_{P Q}\right)^{1 / 3}\right)$. We see that axino masses $\gg m_{3 / 2}$ are indeed possible. An example featuring all these aspects is the superpotential in the fourth entry of the above list for $A, B, C$-type soft terms.

Now we turn to a discussion of the cosmological constraints on the masses of the axino and the saxion. We begin by noticing the fact that self-couplings among the axion supermultiplet 
arise after integrating out the heavy fields in the PQ-sector :

$$
\begin{aligned}
\mathcal{L} & =\left.\sum_{i} v_{i}^{2} \exp \left[q_{i}(\Phi+\bar{\Phi}) / f_{P Q}\right]\right|_{\mathrm{D}-\text { terms }} \\
& \sim\left(1+\frac{\sqrt{2} x}{f_{P Q}} s\right)\left(\frac{1}{2} \partial_{\mu} a \partial_{\mu} a+\frac{1}{2} \partial_{\mu} s \partial_{\mu} s+i \overline{\tilde{a}} \gamma_{\mu} \partial_{\mu} \tilde{a}\right)-\frac{x}{f_{P Q}} \partial_{\mu} a \overline{\tilde{a}} \gamma_{\mu} \tilde{a}+\cdots
\end{aligned}
$$

where $x=\sum_{i} q_{i}^{3} v_{i}^{2} / f_{P Q}^{2}$. In some cases, in particular in a model with superpotential $W=$ $\lambda\left(S S^{\prime}-\mu^{2}\right) Y$ and universal scalar soft masses, $x$ is zero at the Planck scale and receives a contribution $x \sim \lambda^{2} \ln \left(M_{P} / f_{P Q}\right) / 64 \pi^{2}$ when the RG-improved potential at the PQ-scale is considered [13]. Generically, however, $x$ is of order 1 . In this case the self-coupling becomes important since a saxion can decay into two axions faster than e.g., into two gluons. Decayproduced axions do not heat the universe. Therefore the cosmological effect of saxion decay is different from what has been investigated assuming vanishing $x$ [14, 15]. If $x$ is of order 1, a stronger bound on the saxion mass can be expected since the decay-produced axions are not thermalized but red-shifted away. The standard nucleosynthesis constrains the energy density of the universe due to this red-shifted axions to be less than what is contributed by one species of neutrinos at the time of nucleosynthesis. This gives the constraint $m_{s} Y_{s} g_{* D}<T_{D}$ where $T_{D}=0.55 g_{* D}^{-1 / 4} \sqrt{\Gamma M_{P}}$ is the decay temperature of the saxion and $\Gamma=x^{2} m_{s}^{3} / 8 \pi f_{P Q}^{2}$ its decay rate. The relativistic degrees of freedom at $T_{D}$ are counted by $g_{* D}$. We get

$$
m_{s}>2.4 \mathrm{TeV}\left(\frac{f_{P Q} / x}{10^{11} \mathrm{GeV}}\right)^{2}\left(\frac{g_{* D}}{100}\right)^{5 / 2}
$$

This bound on the saxion mass which receives a contribution $O\left(m_{3 / 2}\right)$ from scalar soft masses might be difficult to fulfill for large values of $f_{P Q}$.

Cosmological implications of axinos were first discussed in ref. [6] assuming unbroken $\mathrm{R}^{-}$ parity. The axino mass can be constrained in two ways. First, the axino can be the lightest supersymmetric particle (LSP). Then it should be lighter than a few $\mathrm{keV}$ in order not to overclose the universe. Otherwise, the axino decays into at least one LSP composed out of the neutralinos in the MSSM. In this case, the decay-produced neutralinos tend to overdominate the evergy density of the universe. To avoid this the axino should be heavy enough to decay before the neutralinos decouple. Considering the axino decay into photino plus photon, it was obtained that the axino mass should be bigger than a few $\mathrm{TeV}$ [6]. Then, the axino decay into top quark and scalar top can be allowed. From this decay channel one finds a less restrictive bound

$$
m_{\tilde{a}}>90 \mathrm{GeV}\left(\frac{m_{\chi}^{0}}{40 \mathrm{GeV}}\right)^{2}\left(\frac{f_{P Q} / X_{t}}{10^{11} \mathrm{GeV}}\right)^{2}\left(\frac{174 \mathrm{GeV}}{m_{t}}\right)^{2}\left(\frac{g_{* D}}{100}\right)^{1 / 2} .
$$

Here $X_{t}$ is the $\mathrm{PQ}$-charge of the top quark. We see that a wide range of axino masses between $O(\mathrm{keV})$ and $O\left(10^{2} \mathrm{GeV}\right)$ is excluded. 
Let us now analyze how the above constraints modify if an inflationary expansion is taken into account. The decoupling temperature of axino or saxion is around the range of the reheating temperature $T_{R} \sim 10^{10} \mathrm{GeV}$ which is the maximally allowed value to cure the gravitino problem in supergravity models [16]. If decoupling of the axino occurs before inflation the primordial axino relics are diluted away. The above consideration, then, has to be applied to the regenerated population of axinos. We recall that the axino decoupling is determined by its interactions with gluinos, quarks and anti-quarks via gluon exchange [6]. The axino decouples at the temperature

$$
T_{D} \sim 10^{9} \mathrm{GeV}\left(\frac{f_{P Q}}{10^{11} \mathrm{GeV}}\right)^{2}\left(\frac{0.1}{\alpha_{c}}\right)^{3} .
$$

The regenerated number density per entropy is given by [16]

$$
Y \sim 2 \times 10^{-5}\left(\frac{10^{11} \mathrm{GeV}}{f_{P Q}}\right)^{2}\left(\frac{T_{R}}{10^{10} \mathrm{GeV}}\right)
$$

Depending on the range of the axino mass we can distinguish three cases as follows.

First, for a stable axino, the constraint from overclosure gives the following loose bound on the axino mass in terms of $T_{R}$ :

$$
m_{\tilde{a}}<160 \mathrm{keV}\left(\frac{f_{P Q}}{10^{11} \mathrm{GeV}}\right)^{2}\left(\frac{10^{10} \mathrm{GeV}}{T_{R}}\right) .
$$

Second, an axino with a mass satisfying the lower bound in eq. (22) is still allowed by cosmology. Finally, for an unstable axino with mass between the estimations in eq. (25) and in eq. (22), one gets a bound on the reheating temperature by replacing the axino mass in eq. (25) by the mass of the usual LSP since the decay-products of the axino contain at least one LSP:

$$
T_{R} \lesssim 2 \times 10^{5} \mathrm{GeV}\left(\frac{f_{P Q}}{10^{11} \mathrm{GeV}}\right)^{2}\left(\frac{40 \mathrm{GeV}}{m_{\chi^{0}}}\right) .
$$

This represents a quite stringent bound on $T_{D}$.

In this letter we have analyzed the axino mass in general supergravity models and the cosmological constraints on such models. We have distinguished models with and without additional zero modes in the $\mathrm{PQ}$-sector. For the latter we found the axino mass to be at most of $O\left(m_{3 / 2}\right)$. In the context of an effective approach encoding supersymmetry breaking in soft terms we were able to derive a simple necessary criterion for a small tree level axino mass $\ll m_{3 / 2}$ given in terms of superpotential and soft term properties. For uniform trilinear, bilinear and linear soft couplings $A, B, C$ the criterion is always fulfilled for $A=B=C$ whereas for $A=B \neq C$ or $A \neq B=C$ additional properties of the superpotential had to be required. 
If the global vacuum $(u)$ represents an additional zero mode of the globally supersymmetric theory, i. e. $M u=0$ with the global mass matrix $M$, the relation $A-2 B+C=0$ which admits the standard pattern $B=A-1, C=A-2$ is sufficient for the criterion to hold.

We showed that in models without additional zero modes our criterion is sufficient, i. e. it guarantees a tree level axino mass of at most $m_{\tilde{a}}^{(0)}=O\left(m_{3 / 2}^{2} / f_{P Q}\right)$. In the presence of other zero modes it serves as a good indication for such a small mass but additional conditions (like e. g. $A \neq B$ for models with an $A, B, C$-pattern) are required to have $m_{\tilde{a}}^{(0)}=O\left(m_{3 / 2}^{2} / f_{P Q}\right)$.

From the cosmological point of view axino masses $O\left(m_{3 / 2}^{2} / f_{P Q}\right)=O(\mathrm{keV})$ are very attractive. In this case the axino is the LSP and can contribute a relevant part of the mass in the universe as (warm) dark matter. Though models with such an axino mass can be constructed as we have seen they correspond to very special points in the space spanned by the superpotential and soft term parameters. Moreover, such small masses are not stable under radiative corrections arising from renormalization effects between $M_{P}$ and the $\mathrm{PQ}-$ scale $f_{P Q}$. Taking these effects into account the axino mass will be generically given by $m_{\tilde{a}}^{(1)}=O\left(k m_{3 / 2}\right)$ with $k=\lambda^{2} \ln \left(M_{P} / f_{P Q}\right) / 32 \pi^{2}$ and a typical superpotential coupling $\lambda$. The one-loop contribution from the characteristic coupling of the axino to the Higgs-fields or heavy quarks will be given by $m_{\tilde{a} \text {,loop }} \simeq 3 g^{2} A m_{3 / 2} / 16 \pi^{2}$ which is only relevant in the KVSZ-implementation of the axion. As the LSP the axino has to be lighter than a few $\mathrm{keV}$ which in turn puts a severe limit on the couplings $\lambda$ (and $g$ in the KVSZ-case), typically $\lambda \lesssim 10^{-4}$. If the decoupling temperature of the axino is larger than the reheating temperature of inflation the overclosure bound on the regenerated axino population is weakened resulting in a somewhat weaker bound on $\lambda$, typically $\lambda \lesssim 10^{-3}$. In any case we conclude that a cosmological relevant LSP-axino - though possible in principle - is not very likely to occur : Special models are needed and in addition small couplings have to be chosen in order to avoid a conflict with the overclosure bound.

At this point it should be mentioned that no-scale supergravity models can provide a naturally light axino [9]. Those models are characterized by a special pattern of the soft terms : The only non-vanishing soft terms at the Planck scale are gaugino masses and therefore $A=B=C=0$ at tree level. Applying the above statements a small axino mass results in this case. In fact, a full supergravity calculation shows that the mass vanishes on tree level. Other soft terms for the gauge non-singlets can be generated due to renormalization group effects below the Planck scale in those models. Since the PQ-sector consists of singlets their soft terms are not affected by renormalization effects and the axino (saxion) remains massless [9].

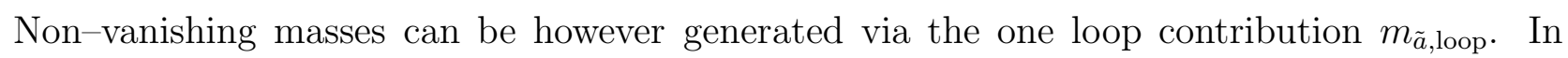
the DFSZ-implementation they are so small that cosmological effects of the axino and the saxino are negligible. This is clearly different in the KVSZ-case. However, the limit on $g$ necessary to keep the axino mass below the overclosure bound will be somewhat weakened with respect to the ordinary case since the trilinear coupling $A$ for $g \Phi Q_{1} Q_{2}$ originates from 
radiative corrections.

As a generic situation we consider models which do not fulfill our criterion for a small tree level axino mass and possess couplings $\lambda=O(1)$. The axino mass in such models is given by $m_{\tilde{a}}=O\left(m_{3 / 2}\right)$ (no additional zero modes) or $m_{\tilde{a}}=O\left(m_{3 / 2} / k\right)$ (additional zero modes). First of all this mass has to be larger than the mass of the ordinary LSP in the MSSM to allow for a decay of axinos. Second, these decays have to occur before the LSP decouples which translates into a bound on $m_{\tilde{a}}$ of typically $m_{\tilde{a}} \gtrsim 100 \mathrm{GeV}$ for $f_{P Q}=10^{11} \mathrm{GeV}$ if the decay channel into top and stop is possible. Otherwise an even stronger bound $m_{\tilde{a}} \gtrsim \mathrm{TeV}$ is required. We see that these generic models are significantly constrained by cosmological considerations, however, a final decision depends on details of the model like the exact axino mass, the sfermion masses, the PQ-scale etc. Therefore it might be interesting to study models which put further constraints on these parameters like e. g. supersymmetric unified models incorporating an axion. For a PQ-scale in the upper half of the allowed range $f_{P Q}>10^{11}$ $\mathrm{GeV}$ the lower bounds on $m_{\tilde{a}}$ and the saxion mass $m_{s}$ (which both increase quadratically with $f_{P Q}$ ) become very stringent and it might be difficult to construct viable models. In this context, having axino masses larger than $m_{3 / 2}$ in models with additional zero modes might be an interesting option.

Acknowledgement. This work was supported by the EC under contract no. SC1-CT910729. We would like to thank K. Choi and D. Matalliotakis for stimulating discussions in the earlier development of this work.

\section{References}

[1] J. E. Kim, Phys. Rev. Lett. 43 (1979) 103; M. A. Shifman, V. I. Vainstein and V. I. Zakharov, Nucl. Phys. B 166 (1980) 4933.

[2] M. Dine, W. Fischler and M. Srednicki, Phys. Lett. B 104 (1981) 199; A. P. Zhitnitskii, Sov. J. Nucl. Phys. 31 (1980) 260.

[3] For reviews see: J. E. Kim, Phys. Rep. 150 (1987) 1; H. Y. Cheng, Phys. Rep. 158 (1988) 1; R. D. Peccei, n "CP Violation", ed. C. Jarlskog (WSPC, Singapore, 1989) 503.

[4] For a natural supersymmetric version of the DFSZ-axion see : E. J. Chun, preprint IC/94/358, hep-ph/9411290 (to appear in Phys. Lett. B).

[5] For a review see : E. Kolb and M. Turner, "The Early Universe" (Addison-Wesley, 1990).

[6] K. Rajagopal, M. S. Turner and F. Wilczek, Nucl. Phys. B 358 (1991) 447. 
[7] K. Tamvakis and D. Wyler, Phys. Lett. B 112 (1982) 451.

[8] J. F. Nieves, Phys. Rev. D 33 (1986) 1762.

[9] T. Goto and M. Yamaguchi, Phys. Lett. B 276 (1992) 103.

[10] E. J. Chun, J. E. Kim and H. P. Nilles, Phys. Lett. B 287 (1992) 123.

[11] P. Moxhay and K. Yamamoto, Phys. Lett. B 151 (1985) 363.

[12] H. P. Nilles, Phys. Rep. 110 (1984) 1.

[13] E. J. Chun, H. B. Kim and A. Lukas, Phys. Lett. B 328 (1994) 346.

[14] J. E. Kim, Phys. Rev. Lett. 67 (1991) 3465.

[15] D. H. Lyth, Phys. Rev. D 48 (1993) 4523.

[16] J. Ellis, J. E. Kim and D. V. Nanopoulos, Phys. Lett. B 145 (1984) 181. 